\title{
Remerciements aux lecteurs arbitres
}

Natures Sciences Sociétés tient à remercier l'ensemble des personnes, dont les noms suivent, qui ont accepté avec dévouement et déontologie d'évaluer les manuscrits soumis à la revue en 2018. Ils ont éclairé la prise de décision du comité de rédaction et ont ainsi contribué par leurs critiques exigeantes à la sélection des manuscrits soumis et à la qualité des articles publiés.

Barbier Marc
Bataille Nicolas
Bonin Muriel
Bonnaud Laure
Bouba-Olga Olivier
Brédif Hervé
Cartier Stéphane
Cerf Marianne
Charvolin Florian
Colonna Paul
Compagnone Claude
Corneloup Jean
Daviron Benoît
Deffuant Guillaume
Dobigny Laure
Doré Thierry
Doussan Isabelle
Germaine Marie-Anne
Girard Nathalie
Gramaglia Christelle
Granjou Céline
Flanquart Hervé
Fournier Stéphane
Hainzelin Étienne
Héritier Stéphane
Karsenty Jean-Paul
Jean Bruno
Lallau Benoît
Cang

Larrère Catherine

Laurans Yann

Le Moigne Jean-Louis

L'Hostis Monique

Maris Virginie

Mathieu Nicole

Maublanc Marie-Line

Michelot Agnès

Micoud André

Monsaingeon Baptiste

Morel-Journel Christelle

Mounet Jean-Pierre

Nahrat Stéphane

Piveteau Vincent

Plumecocq Gaël

Quénol Hervé

Rocle Nicolas

Rodet Guy

Roussel Fabien

Servais Véronique

Soulé Bastien

Touzard Jean-Marc

Varenne Franck

Vatin François

Waser Anne-Marie

Weisbein Julien

Zelem Marie-Christine 